\title{
Weight-loss maintenance 1, 2 and 5 years after successful completion of a weight-loss programme
}

\author{
Michael R. Lowe ${ }^{1 *}$, Tanja V. E. Kral ${ }^{2}$ and Karen Miller-Kovach ${ }^{3}$ \\ ${ }^{1}$ Drexel University, Department of Psychology, 245 N 15th Street, Mail Stop 626, Philadelphia, PA 19102, USA \\ ${ }^{2}$ University of Pennsylvania School of Medicine, Philadelphia, Pennsylvania, USA \\ ${ }^{3}$ Weight Watchers International, Woodbury, New York, USA
}

(Received 19 January 2007 - Revised 9 October 2007 - Accepted 9 October 2007)

The objective was to assess weight-loss maintenance in individuals who successfully completed a commercial weight-loss programme at multiple sites in the United States. A total of 699 lifetime members of Weight Watchers represented the national sample and 217 additional lifetime members served as an oversample. Lifetime members were asked to self-report their current weight 1,2, and 5 years after they had successfully completed the programme. Additional lifetime members served as an oversample whose weights were measured. The discrepancy between reported and measured weight in the oversample was used to adjust the self-reported weights of the national sample. Seventy-one percent of participants were middle-aged or older and $95.3 \%$ were female; their mean starting BMI was 27.6 (SD 3.6 ) kg/m $\mathrm{m}^{2}$. The percentage of Weight Watchers lifetime members who maintained at least $5 \%$ of their weight loss 1,2 and 5 years after successful completion of the programme was $79 \cdot 8,71 \cdot 0$, and $50 \cdot 0$, respectively. The percentage of participants who remained below their goal weight 1,2 and 5 years after completion of the programme was $26 \cdot 5$, $20 \cdot 5$, and 16.2, respectively. Results obtained with this group of successful Weight Watchers members are not directly comparable to those obtained with clinical samples of obese dieters because the current sample comprises only the most successful Weight Watchers participants. However, these results provide further evidence that maintenance of weight loss in those who successfully lose weight in one commercial weight-loss programme is more feasible than data from clinical populations have suggested.

Obesity: Weight loss: Dieting: Weight-loss maintenance

With the steady increase in the prevalence of overweight and obesity in the United States and worldwide ${ }^{(1)}$ more and more individuals are seeking help from weight-loss programmes. Nearly all randomized trials of lifestyle programmes for weight loss have been conducted in clinical (university and medical) settings. However, the vast majority of people seeking assistance with weight loss do so through commercial weight-loss programmes. It has been suggested that those who seek obesity treatment in medical settings may suffer from more psychopathology and binge eating than those who do not ${ }^{(2,3)}$. Little is known about whether the long-term results of randomized, controlled research conducted in university or medical settings ${ }^{(4)}$ are applicable to the much greater number of people who join a weight-loss programme that is available in their community.

Weight Watchers is a widely available, lifestyle-based weight-loss programme. For more than two decades, Weight Watchers has periodically assessed and reported weight-loss maintenance among its successful members. Christakis \& Miller-Kovach ${ }^{(5)}$ reported on a telephone survey conducted among 1200 Weight Watchers members who had successfully reached their goal weight during the period 1981 to 1992. Based entirely on self-reported data, 97, 80, and $37 \%$ of those surveyed indicated that they had stayed within $2.3 \mathrm{~kg}$ $(5 \mathrm{lb})$ of their weight goal at 1,2 , and $5+$ years.
In 2001, Lowe and colleagues examined weight-loss maintenance among Weight Watchers participants who had reached their goal weights 1-5 years previously (covering the period 1992 to 1996). A sample of 1002 who became Weight Watchers lifetime members (by reaching their goal weights and maintaining them for at least 6 weeks) were selected from a pool of lifetime members from various geographical areas in the United States. These lifetime members completed a phone-based survey to assess their level of long-term weight-loss maintenance. In addition, a second sample (oversample; $n$ 259) was recruited from the same pool of lifetime members and weighed in-person to develop an adjustment factor for the self-reported weights obtained via the phone survey. The results of this study showed that, based on adjusted body weights, participants regained 31.5 , 53.7 and $76.5 \%$ of their original weight losses at 1-, 2- and 5-year follow-ups, respectively.

As Tsai \& Wadden pointed out in their review on commercial weight-loss programmes ${ }^{(6)}$ there is a need to assess the effectiveness of these programmes in terms of both weight loss and weight-loss maintenance. The purpose of this paper is to present new data on weight-loss maintenance 1, 2 and 5 years following successful weight loss among Weight Watchers members. Data were collected on former Weight Watchers members who lived in a number of widely dispersed areas of 
the United States. A shortcoming of the Lowe et al. ${ }^{(7)}$ study was that both the starting weight (weight at joining the programme) and the goal weight reached at the end of the programme were recalled by members one or more years later, a procedure which left the accuracy of these self-reported weights open to question. This weakness was rectified in the present study because both the start and the goal weights were measured by Weight Watchers when participants were in the programme. Information about follow-up body weights was collected 1,2 and 5 years following the attainment of goal weight using the same procedures as in the earlier study. These new long-term follow-up results are reported here and are compared to similar results described in the $2001 \operatorname{report}^{(7)}$.

\section{Methods}

\section{Programme}

The Weight Watchers programme is built on four fundamental components: (1) a food plan, (2) an activity plan, (3) a behaviour modification plan which is based on cognitive restructuring, and (4) group support. The food plan consists of a nutritionally balanced diet which is designed to induce a moderate energy deficit and a weight loss of up to $0.9 \mathrm{~kg} /$ week The programme also contains a structured activity plan which is designed to progressively increase moderate exercise among participants to increase daily energy expenditure. The overall goal of the activity plan at the start of the programme is to reduce sedentary behaviour among participants. This goal progresses through the course of the programme to promote $60 \mathrm{~min}$ of moderate activity per day during the weight-loss maintenance phase. During weekly meetings, which are led by trained staff who have attained lifetime status and serve as role models, participants engage in group support, are provided with educational information and materials, and are privately weighed.

As part of the programme, each member determines his or her individual goal weight. The goal weight must be at least $2.3 \mathrm{~kg}(5 \mathrm{lb})$ below the member's initial weight when joining the programme and typically falls within the BMI range for normal weight $\left(20-25 \mathrm{~kg} / \mathrm{m}^{2}\right)$. The majority of members typically choose a goal weight which is equivalent to a BMI of $25 \mathrm{~kg} / \mathrm{m}^{2}$. If desired, a member can choose a goal weight at a BMI of $>25 \mathrm{~kg} / \mathrm{m}^{2}$ and still be eligible for lifetime status provided that a qualified health-care professional provides written confirmation that the chosen weight goal is a healthy weight for that individual. Once a member reaches his or her prescribed goal weight over the course of the programme and successfully completes the 6-week maintenance phase of the programme, Weight Watchers confers lifetime member status to that individual. Lifetime members are exempt from paying the registration fee, are asked to weigh-in monthly at a meeting (to encourage continued contact and support) and receive all services at no cost as long as they stay within $0.9 \mathrm{~kg}$ of their goal weight.

\section{Procedures}

Participants in this study had attained lifetime member status from Weight Watchers 1, 2 or 5 years before the present study was initiated. All participants resided in the United States.
To locate these lifetime members, a zip code analysis was conducted to identify and stratify a national sample. That is individuals to be contacted were selected in proportion to their representation in different regions of the country. A telephone survey was administered by an independent research firm to a total of 1008 lifetime members randomly selected from all lifetime members eligible for this study. Therefore, the geographical distribution of the lifetime members generally reflected their distribution across the United States. Members were selected from four different national regions (East, West, South, Central); the number of participants from each region was equal. The phone-based survey interview was designed to assess long-term maintenance of weight loss and pertinent member demographics. As part of the survey participants were asked to verify the date at which their lifetime member status was conferred to them by Weight Watchers and to report their current weight (in lb). Participants' weight when they joined Weight Watchers and the goal weight at which they achieved their lifetime member status were taken from Weight Watchers' records. These records were retained by most but not all Weight Watchers' centres, but their availability or non-availability was based on practices followed by local Weight Watchers' centres and had nothing to do with this study.

The total national sample consisted of 1008 participants. To be included in the analyses national sample participants needed to have a valid start weight, lifetime goal weight, and current weight. These weights, measured with shoes removed, were available from 699 participants of this national sample. All analyses were based on this subgroup of participants. Furthermore, 663 additional participants were recruited (at the same time as were national sample participants) from four geographically dispersed metropolitan areas (Atlanta, Baltimore, Los Angeles, and Minneapolis) which were close to research sites operated by the survey research firm who collected the data. These additional participants served as an oversample for the purpose of developing an adjustment factor for self-reported weights in the national sample. Oversample participants completed the same phone-based survey interview as did participants of the national sample. They were also asked if they would be willing to participate in an additional survey to be conducted in a nearby facility (they were not told that they would be weighed as part of the additional survey). An incentive of \$25 was offered to encourage participation in the additional survey. Those subjects who were willing to participate were contacted by the local market research facility to schedule an appointment. During this visit participants were weighed to the nearest $0.09 \mathrm{~kg}$ on a digital scale (Tanita Corporation, Skokie, IL, USA), in light clothing with shoes off. Out of the total oversample 225 participants (about one-third of all those contacted) agreed to make an onsite visit and were weighed during their appointment (onsite oversample). Data from 217 of these participants (seven outliers were removed; see later) were used to develop the adjustment factor.

\section{Statistical analyses}

All statistical analyses in the study were performed using SPSS data analysis software (version 12.0 for Windows; 
SPSS Inc., Chicago, IL, USA). Data were expressed as percentages or as means with SD. Contrasts between means were assessed by Student's $t$ tests. Pearson correlation coefficients were used to determine the relationship between continuous variables. Results were considered statistically significant at $P<0 \cdot 05$.

\section{Results}

\section{Sample characteristics}

The demographic characteristics of the national sample are displayed in Table 1. The majority of the national sample was female $(95.3 \%)$, married $(82.1 \%)$ and employed $(55.2 \%)$. Most participants were above the age of 45 years $(71.3 \%)$ and had family incomes over $\$ 40000 /$ year $(76.2 \%)$. These characteristics generally mirror the make-up of the typical Weight Watchers member, who is relatively well-off financially and has at least some college education. Data on the ethnicity of the sample were not collected in the current study. It is likely, however, that the current sample closely resembled the sample of the previous study ${ }^{(7)}$.

Subjects from both samples (onsite oversample and national sample) were similar in demographic characteristics. Furthermore, there was no significant difference $(P=0 \cdot 61)$ in self-reported BMI calculated from subjects' self-reports of height and weight between subjects in the national sample $\left(24.1\right.$ (SD 2.4) $\mathrm{kg} / \mathrm{m}^{2}$ ) and subjects in the onsite sample
(23.9 (SD 2.60) kg/m²) (with outliers removed; see later). Oversample participants who did not come in to be weighed were not significantly different $(P=0.74)$ in self-reported BMI (24.1 (SD 2.7) $\mathrm{kg} / \mathrm{m}^{2}$ ) from those who were weighed at one of the research facilities $\left(23.9(\mathrm{SD} 2.6) \mathrm{kg} / \mathrm{m}^{2}\right.$ ).

\section{Comparison between included and excluded national sample participants}

Excluded national sample participants for whom measured start BMI ( $n$ 104; start BMI = 28.2 $($ SD 4.05$\left.) \mathrm{kg} / \mathrm{m}^{2}\right)$ and goal weights $(n 115$; goal weight $=63.7(\operatorname{SD} 8.49) \mathrm{kg})$ were available did not differ on those variables from national sample participants ( $n$ 699; start $\mathrm{BMI}=27.6(\mathrm{SD} 3.60) \mathrm{kg} / \mathrm{m}^{2}$, $P=0 \cdot 14$; goal weight $=64.0($ SD 7.0$) \mathrm{kg}, P=0.75)$ who were included in the analyses, respectively. The current BMI (adjusted) from excluded national sample participants $\left(n=309\right.$; current $\left.\mathrm{BMI}=25.7(\mathrm{SD} 3 \cdot 22) \mathrm{kg} / \mathrm{m}^{2}\right)$, however, was significantly higher $(P=0 \cdot 001)$ than was that of included national sample participants (24.6 (SD 2.4) $\mathrm{kg} / \mathrm{m}^{2}$ ).

\section{Adjustment factor}

In order to correct for any bias in self-reported weights an adjustment factor was computed using self-reported and measured weights from oversample participants ( $n$ 217). The adjustment factor represented the average discrepancy

Table 1. Demographic characteristics of national and onsite samples

\begin{tabular}{|c|c|c|}
\hline Variable & National sample ( $n$ 699) & Onsite sample $(n 217)$ \\
\hline \multicolumn{3}{|l|}{ Gender (\%) } \\
\hline Female & $95 \cdot 3$ & $92 \cdot 6$ \\
\hline Male & 4.7 & 7.4 \\
\hline \multicolumn{3}{|l|}{ Age (\%) } \\
\hline Under 18 & 0.4 & NA \\
\hline $18-24$ & 0.4 & 0.5 \\
\hline $25-29$ & 2.5 & 0.9 \\
\hline $30-34$ & $6 \cdot 2$ & 4.2 \\
\hline $35-44$ & $19 \cdot 1$ & $26 \cdot 7$ \\
\hline $45-54$ & 28.4 & 29.1 \\
\hline $55-64$ & 21.5 & $28 \cdot 2$ \\
\hline 65 and over & 21.4 & $10 \cdot 3$ \\
\hline Start weight $(\mathrm{kg})$ & $74.9(\mathrm{SD} 11.7)$ & 73.4 (SD 10.9) \\
\hline Start BMI $\left(\mathrm{kg} / \mathrm{m}^{2}\right)$ & 27.6 (SD 3.6) & 26.4 (SD 3.1) \\
\hline \multicolumn{3}{|l|}{ Marital status (\%) } \\
\hline Single & $7 \cdot 0$ & $8 \cdot 8$ \\
\hline Married & $82 \cdot 1$ & 77.4 \\
\hline Separated or divorced & 3.4 & 3.7 \\
\hline Widowed & 5.9 & $4 \cdot 1$ \\
\hline Employment status (outside home) (\%) & $55 \cdot 2$ & $64 \cdot 6$ \\
\hline Full-time & $73 \cdot 3$ & $73 \cdot 6$ \\
\hline Part-time & $26 \cdot 7$ & $26 \cdot 4$ \\
\hline \multicolumn{3}{|l|}{ Annual income (\%) } \\
\hline Under $\$ 20000$ & $3 \cdot 7$ & \\
\hline$\$ 20000$ to under $\$ 25000$ & $5 \cdot 2$ & \\
\hline$\$ 25000$ to under $\$ 30000$ & $5 \cdot 2$ & \\
\hline$\$ 30000$ to under $\$ 35000$ & 4.3 & \\
\hline$\$ 35000$ to under $\$ 40000$ & 5.4 & NA \\
\hline$\$ 40000$ to under $\$ 50000$ & 11.9 & \\
\hline$\$ 50000$ to under $\$ 75000$ & $21 \cdot 2$ & \\
\hline$\$ 75000$ and over & $43 \cdot 1$ & \\
\hline
\end{tabular}

NA, data for this category were not available. 
between measured and self-reported weight (i.e. (difference between measured weight and self-reported weight)/(selfreported weight)). Six women and one man with extreme values ( $>3$ SD from the mean) were excluded from further analysis on the presumption that their self-reported values were due to key-punching or transcription errors. The discrepancy between self-reported and measured weights in these individuals ranged from $+49.5 \mathrm{~kg}$ (self-reported weight $>$ measured weight) to $-20.0 \mathrm{~kg}$ (self-reported weight $<$ measured weight). The mean difference in self-reported and measured body weight was -1.52 (SD 1.54) kg. Excluding the seven outliers, the average degree of underreporting in the oversample was 2.29 (SD 2.30) \%. All results are reported as adjusted values. Adjusted weights were derived by multiplying self-reported weights by 1.0229 .

\section{Maintenance of weight losses}

Weight maintenance was examined for the 699 participants of the national sample who had reached goal weight 1,2 and 5 years previously. Table 2 illustrates the adjusted weight regain in the following ways: (1) the amount of weight regained $(\mathrm{kg}),(2)$ the percentage of weight loss regained, (3) the percentage of participants who remained within $2.3 \mathrm{~kg}(5 \mathrm{lb})$ of goal weight, (4) the percentage of participants who maintained a weight loss of $\geq 5 \%$ of initial body weight, (5) the percentage of participants who maintained a weight loss of at least $10 \%$ of initial body weight, (6) the percentage of participants who were still below their initial weight when they joined Weight Watchers, and (7) the percentage of participants who were below their goal weight.

Mean weight loss of participants in the national sample (n 699) to reach goal weight was 10.9 (SD 7.6) kg. Participants' mean start weight was 74.9 (SD 11.7) $\mathrm{kg}$; their mean goal weight was 64.0 (SD 7.0) kg. During years 1,2 and 5, subjects regained on average $2 \cdot 16$ (SD 4.5), 3.45 (SD 5.1), and 4.70 (SD 5.9 ) $\mathrm{kg}$, respectively, or $23.8,37.5$, and $62.4 \%$ of their initial weight loss. The percentage of participants who maintained $\geq 5 \%$ of weight loss during years 1,2 and 5 of the follow-up period amounted to $79 \cdot 8,71 \cdot 0$, and $50.0 \%$, respectively. The percentage of participants who remained below their initial weight (i.e. before weight loss) was also high at $94 \cdot 1,90 \cdot 7$, and $75 \cdot 0$ during years 1,2 and 5 respectively.

Fig. 1 illustrates the adjusted weight regain among national participants from the 2003 study in comparison with the 1997 study.
Associations between weight underestimation and current weight/weight-loss regain among onsite oversample participants

Among onsite oversample participants the degree of bodyweight underestimation was significantly correlated with measured $(r 0.274, P<0.01)$ but not (unadjusted) self-reported weight $(r 0 \cdot 107, P=0 \cdot 115)$; thus more-overweight participants underestimated their weight to a greater degree than less-overweight participants. However, because there was no difference in BMI between the national and onsite oversample (see earlier), this relationship would not be expected to produce any bias when the adjustment factor was used to adjust the self-reported weights of the national sample. Also, based on adjusted results in the oversample, the degree of underestimation of body weight was not significantly correlated with weight regain $(r-0 \cdot 015, P=0 \cdot 844)$.

\section{Discussion}

The current study closely replicated an earlier investigation of weight-loss maintenance in Weight Watchers lifetime members 1-5 years after lifetime membership had been achieved $^{(7)}$. The current study used the same methodology to examine long-term follow-up of weight-loss maintenance in a nationally representative sample of lifetime members who reached goal weight 1,2 or 5 years previously. Based on the Institute of Medicine ${ }^{(8)}$ criterion for successful weightloss maintenance of sustaining at least a $5 \%$ weight loss, the current study found that $79 \cdot 8,71 \cdot 0$, and $50.0 \%$ successfully maintained a weight loss achieved 1,2 or 5 years previously, respectively. It is important to note that the improved results in the current study occurred despite the fact that Americans generally continued to gain weight during the 5 -year period under study ${ }^{(1)}$. A sustained weight loss of $\geq 5 \%$ also produces medically significant improvements in comorbidities associated with obesity ${ }^{(8-11)}$. Therefore, although medical outcomes were not assessed, the weight-maintenance results described here would likely have been associated with important improvements in medical risk factors associated with obesity.

One striking finding in this study was that weight-loss maintenance over the course of 5 years following the completion of the programme was substantially improved compared with findings from the previous report of long-term weightloss maintenance in Weight Watchers lifetime members ${ }^{(7)}$.

Table 2. Adjusted weight regain among national sample participants ( $n$ 699)

\begin{tabular}{|c|c|c|c|c|c|c|}
\hline & \multicolumn{2}{|c|}{$\begin{array}{l}\text { 1-year follow-up } \\
\text { ( } n \text { 272) }\end{array}$} & \multicolumn{2}{|c|}{$\begin{array}{l}\text { 2-year follow-up } \\
\text { ( } n \text { 279) }\end{array}$} & \multicolumn{2}{|c|}{$\begin{array}{l}\text { 5-year follow-up } \\
\text { (n 148) }\end{array}$} \\
\hline & Mean & SD & Mean & SD & Mean & SD \\
\hline Weight regain $(\mathrm{kg})$ & $2 \cdot 16$ & 4.5 & 3.45 & $5 \cdot 1$ & $4 \cdot 70$ & 5.9 \\
\hline Percentage of weight loss regained & $23 \cdot 8$ & $53 \cdot 3$ & 37.5 & $65 \cdot 6$ & $62 \cdot 4$ & $110 \cdot 7$ \\
\hline Percentage of participants who remained within $5 \mathrm{lb}(2.27 \mathrm{~kg})$ of goal weight & \multicolumn{2}{|c|}{$56 \cdot 3$} & \multicolumn{2}{|c|}{$44 \cdot 8$} & \multicolumn{2}{|c|}{37.2} \\
\hline Percentage of participants who maintained $\geq 5 \%$ weight loss & \multicolumn{2}{|c|}{79.8} & \multicolumn{2}{|c|}{71.0} & \multicolumn{2}{|c|}{$50 \cdot 0$} \\
\hline Percentage of participants who maintained $\geq 10 \%$ weight loss & \multicolumn{2}{|c|}{$54 \cdot 4$} & \multicolumn{2}{|c|}{$45 \cdot 2$} & \multicolumn{2}{|c|}{$28 \cdot 4$} \\
\hline Percentage of participants who remained below their initial weight & \multicolumn{2}{|c|}{$94 \cdot 1$} & \multicolumn{2}{|c|}{$90 \cdot 7$} & \multicolumn{2}{|c|}{$75 \cdot 0$} \\
\hline Percentage of participants who remained below their goal weight & \multicolumn{2}{|c|}{26.5} & \multicolumn{2}{|c|}{20.5} & \multicolumn{2}{|c|}{$16 \cdot 2$} \\
\hline
\end{tabular}




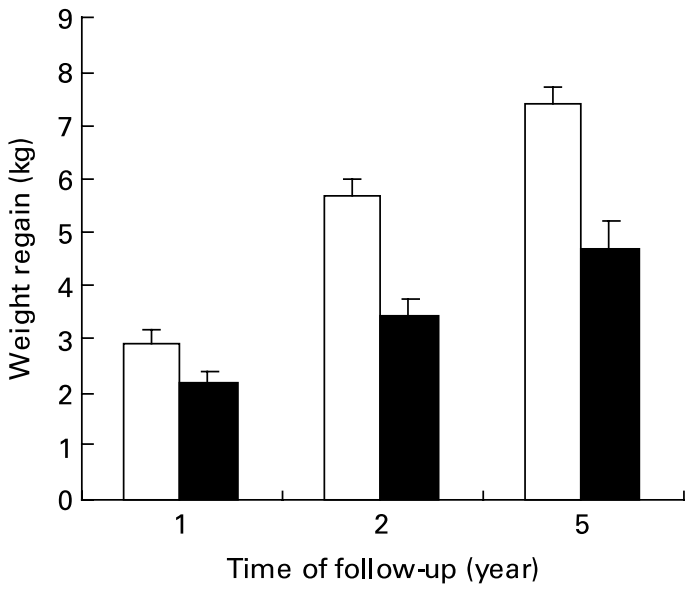

Fig. 1. Adjusted weight regain (mean standard error) among national participants from the $1997(\square)$ and 2003 (ם) studies. Values are means with standard error indicated by vertical bars.

Participants in the previous study regained an average of $2 \cdot 9$, $5 \cdot 7$, and $7 \cdot 4 \mathrm{~kg} 1,2$ and 5 years after reaching goal weight; the comparable figures in the present study were $2.2,3.5$ and $4.7 \mathrm{~kg}$. One reason for the improved findings in the current study may be that measured, instead of self-reported, data were available for both the starting weight and the weight achieved at the end of the weight-loss period (i.e., goal weight). In the previous study, if participants' self-reported starting weights were underestimated and/or their goal weights were overestimated, this would produce percentage weightregain figures that were higher than they really were. In addition, in the current study we removed outliers (those participants who deviated from their measured weight by $>3$ SD from the mean based on their self-reported weight) before computing the adjustment factor, a procedure which had not been previously undertaken. Taken together, the present results appear to be a truer estimate of lifetime members' long-term weight maintenance, especially because they are based on measured starting and goal weights.

The improvement in long-term maintenance in this report compared to the previous one was substantial and raises the question of what might have accounted for this improvement. It is impossible to determine definitively the reasons for the better outcome, but it is worth noting that the Weight Watchers programme underwent a major change between the period covered by the first publication $^{(7)}$ and the current one. During the former period, members followed an exchange-type diet (e.g. eat three proteins, four breads, two fruits). During the period examined here, members followed the POINTS ${ }^{\circledR}$ Weight-Loss System. The System assigns a POINTS value calculated based on a formula that includes energy, fat, and fibre contents per serving. Members have a daily POINTS Target based on individual factors (e.g. age, gender). By tracking their intake with simple, single-digit numbers, the weight-loss process is simplified while encouragement is provided to make lower-fat, higherfibre food choices. Although both methods are based on the same energy deficit, it may be that members found the newer system easier to adhere to and therefore maintained their weight losses better. As mentioned earlier, there are limited data available on long-term maintenance in individuals who successfully completed a commercial weight-loss programme. Grodstein et al. ${ }^{(12)}$ conducted a study in which 192 participants, who completed a commercial weight-loss programme (Optifast; $422-1004 \mathrm{kcal} / \mathrm{d}$ depending on weight status) 3 years earlier, self-reported their body weight at follow-up. The results of this study showed that $57 \%$ of participants maintained $\geq 5 \%$ of the weight loss. Because these follow-up results were based on self-report, they likely overestimate the actual level of weight-loss maintenance.

Gosselin \& Cote $^{(13)}$ conducted a study in which they collected information about weight maintenance in women 2-11 years after their participation in a commercial weightloss programme (Mincavi; balanced deficit diet, $1400 \mathrm{kcal} / \mathrm{d}$ for women, $1800 \mathrm{kcal} / \mathrm{d}$ for men) in the province of Quebec, Canada. Data were based on self-reported weights for the sample as a whole, and verified body weights via existing records for a subsample (n 31) of participants. A correction factor of $2.9 \%$ was applied to subjects' self-reported weight to adjust for underreporting. According to the results of this study, the percentage of women who successfully maintained $\geq 5 \%$ of their initial weight loss after $2,3,3,5-6,7-8$, and 9-11 years of participating in a commercial weight-loss programme was $43.6,33.3,23.8,38.2,29.4$ and $19.6 \%$, respectively.

The weight-maintenance results reported here are far better than those reported in randomized-controlled trials of lifestyle-change programmes. Among those completing such trials, at least one-third of lost weight is regained after 1 year, approximately two-thirds is regained after 2 years, and almost all weight lost is regained after 5 years ${ }^{(4)}$. However, the results of the current study are not directly comparable with those from controlled trials for several reasons. Lifetime members represent the most successful subgroup of all those who initially join Weight Watchers, whereas the majority of those who join randomized clinical studies complete the programme and are included in the data analyses. It is possible that if only the most successful weight losers from clinical trials were examined at follow-up, their long-term outcomes might be comparable to those reported here. Individuals who seek treatment in universities or medical centres may also be more likely to suffer from psychopathology and binge eating than those attending programmes in the community, factors that may affect ability to lose weight and/or maintain the loss. Furthermore, participants were mostly women, primarily middle-aged, married, employed and had a relatively high income. The present results may not be applicable to samples which differ from the present sample on one or more of these variables.

In addition, the degree of overweight in lifetime members when they began Weight Watchers was considerably lower $\left(\mathrm{BMI}=27.59 \mathrm{~kg} / \mathrm{m}^{2}\right)$ than the average of people enrolling in clinical trails (whose BMI are typically in the $30-35 \mathrm{~kg} / \mathrm{m}^{2}$ range). When adjusting for starting BMI in the present sample, the results showed that starting BMI only accounted for a small amount of variance in outcome. Also, the correlation between starting BMI and weight loss in the current data was negligible $(r$ 0.09). Although these results suggest that the positive outcomes experienced by the lifetime members cannot be explained by their relatively lower body mass, it is still possible that as a group lifetime members' 
impressive weight maintenance was due in part to the fact that their BMI were on average considerably lower than participants in past clinical trials. Excluded national sample participants, relative to those included in the analyses, showed significantly higher current BMI. This result, along with the positive correlation found between weight regain and current BMI, suggests that the weight-regain figures presented in this paper may represent a slight overestimate of the success in weight maintenance of lifetime members of Weight Watchers. Also, since the majority of the sample consisted of middle-class females between the ages of 40 and 65 years, the applicability of the outcomes of this study to a broader population may be limited. Finally, we note that only about one-third of those invited to be part of the oversample agreed to do so. It is possible that this could have biased the sample toward individuals who reported their weight more accurately, since those who were less accurate may have been reluctant to be weighed. Arguing against this interpretation, however, is evidence that the size of the underreporting error we found here is similar to that found in overweight samples in past studies ${ }^{(14,15)}$

We also note that we had no information on whether the lifetime members sought additional treatment during the follow-up periods. Given their success in losing weight initially, this may have occurred and could have contributed to the observed results at follow-up.

In conclusion, the positive outcomes of this long-term follow-up of weight-loss maintenance in participants who successfully completed a commercial weight-loss programme several years prior suggest that impressive levels of long-term maintenance of initial weight-loss may be more feasible than is generally assumed. Since the current study was uncontrolled, however, these results cannot be directly attributed to the enduring effects of the weight-loss programme. Given the paucity of outcome data on commercial weight-loss programmes ${ }^{(6)}$, however, the current data provide important new information on the long-term outcomes of those individuals who reach their goal weights in at least one such programme.

\section{Acknowledgements}

M. R. L. and T. V. E. K. served as consultants to Weight Watchers for the preparation of this manuscript. M. R. L. also serves on the Scientific Advisory Board of Weight Watchers. He advised Weight Watchers and the independent research firm on the design of the study, oversaw data analyses and helped write the manuscript. T. V. E. K. helped analyse the data and write the manuscript. K. M.-K is employed by Weight Watchers.
She helped design the survey and provided feedback on the manuscript.

\section{References}

1. Flegal KM, Carroll MD, Ogden CL \& Johnson CL (2002) Prevalence and trends in obesity among U.S. adults, 19992002. J Am Med Assoc 288, 1723-1727.

2. Brownell KD \& Rodin J (1994) The dieting maelstrom: is it possible and advisable to lose weight. Am Psychol 49, 781-791.

3. Fitzgibbon ML, Stolley MR \& Kirschenbaum DS (1993) Obese people who seek treatment have different characteristics than those who do not seek treatment. Health Psychol 12, $342-345$.

4. Wadden TA, Sarwer DB \& Berkowitz RI (1999) Behavioural treatment of the overweight patient. Baillieres Best Pract Res Clin Endocrinol Metab 13, 93-107.

5. Christakis G \& Miller-Kovach K (1996) Maintenance of Weight Goal among Weight Watchers Lifetime Members. Nutr Today 31, 29-31.

6. Tsai AG \& Wadden TA (2005) Systematic review: an evaluation of major commercial weight loss programs in the United States. Ann Intern Med 4, 56-66.

7. Lowe MR, Miller-Kovach K \& Phelan S (2001) Weight-loss maintenance in overweight individuals one to five years following successful completion of a commercial weight loss program. Int J Obes Relat Metab Disord 25, 325-331.

8. Institute of Medicine (1995) Weighing the Options: Criteria for Evaluating Weight Management Programs. Washington, DC: US Government Printing Office.

9. Noakes M \& Clifton PM (2000) Weight loss and plasma lipids. Curr Opin Lipidol 11, 65-70.

10. Pi-Sunyer FX (1996) A review of long-term studies evaluating the efficacy of weight loss in ameliorating disorders associated with obesity. Clin Ther 18, 1006-1035.

11. Valsamakis G, McTernan PG, Chetty R, Al Daghri N, Field A, Hanif W, Barnett AH \& Kumar S (2004) Modest weight loss and reduction in waist circumference after medical treatment are associated with favorable changes in serum adipocytokines. Metabolism 53, 430-434.

12. Grodstein F, Levine R, Troy L, Spencer T, Colditz GA \& Stampfer MJ (1996) Three-year follow-up of participants in a commercial weight loss program. Can you keep it off? Arch Intern Med 156, 1302-1306.

13. Gosselin C \& Cote G (2001) Weight loss maintenance in women two to eleven years after participating in a commercial program: a survey. BMC Womens Health 1, 2.

14. Visscher TL, Viet AL, Kroesbergen IH \& Seidell JC (2006) Underreporting of BMI in adults and its effect on obesity prevalence estimations in the period 1998 to 2001. Obesity 14, 2054-2063.

15. McAdams MA, Van Dam RM \& Hu FB (2007) Comparison of self-reported and measured BMI as correlates of disease markers in US adults. Obesity 15, 188-196. 